\section{Akuter Myokardinfarkt: Ausschlussdiagnose in einer Stunde}

\author{
Ein neuer Algorithmus für die Troponinbestimmung bei Patienten mit Ver- \\ dacht auf Myokardinfarkt kann die Notfallversorgung beschleunigen. Er lie- \\ fert eine Ausschlussdiagnose mit hoher Genauigkeit bereits innerhalb einer \\ Stunde.
}

B isher empfahlen die kardiologischen Leitlinien für den Nicht-ST-Hebungsinfarkt (NSTEMI) in Europa, bei Patienten mit akutem Brustschmerz direkt bei Aufnahme und noch einmal drei Stunden später den kardialen Biomarker Troponin zu bestimmen, bevorzugt mit hoch sensitiven Tests. So werden heute in den meisten Kliniken jene Patienten identifiziert, bei denen wahrscheinlich ein NSTEMI vorliegt und eine Katheteruntersuchung daher nötig ist.

PD Dr. Dirk Westermann präsentierte beim Europäischen Kardiologenkongress in London die Ergebnisse der BACC („Biomarkers in Acute Cardiovascular Care")-Studie, die einen anderen Weg ging. Die Kardiologen verglichen bei 1.045 Patienten mit akuten Brustschmerzen das in Deutschland gängigste Vorgehen, bei dem hoch sensitives Troponin T (hsTNT) sofort und nach drei Stunden gemessen wird, mit einer Messung des hoch sensitiven Troponin I (hsTNI) sofort und nach nur einer Stunde. Referenz war die klinische Enddiagnose unter Einbeziehung aller Informationen.

\section{Sehr niedrigen Grenzwert gewählt}

Dabei wurde für eine optimale Sensitivität bei der Ausschlussdiagnostik („rule-out“) für beide Messungen ein sehr niedriger Grenzwert von $6 \mathrm{ng} / \mathrm{l}$ gewählt. Auf Basis dieses Grenzwerts hätten nach einer Stunde knapp $40 \%$ der Patienten entlassen werden können.

Dabei berechneten die Hamburger Kardiologen einen negativ-prädiktiven Wert von 99,7\% (Sensitivität 99,1\%) für den Nicht-St-Hebungsinfarkt Typ 1. Das heißt: Maximal einer von 100 Patienten wird entlassen, obwohl ein NSTEMI vorliegt. Das war nicht schlechter als beim Drei-Stunden-Algorithmus auf Basis des hsTNT.

\section{Validierung an zwei Studien}

Die hsTNI-Daten seien mittlerweile an zwei weiteren, unabhängigen Kohorten validiert worden, so Westermann, nämlich an den Teilnehmern der Studien APACE und ADAPT. In diesen Validierungskohorten lag der negativ-prädiktive Wert bei 99,2\% und 99,7\%. Damit könne der auf hsTNI mit einem Grenzwert von $6 \mathrm{ng} / \mathrm{l}$ basierende Ein-StundenAlgorithmus klinisch zur Ausschlussdiagnostik eingesetzt werden.

Am Universitätsklinikum Hamburg Eppendorf soll er jetzt eingeführt werden. Die Hamburger stehen damit auf dem Boden der in London vorgestellten Neufassung der europäischen NSTEMILeitlinie, die einen Ein-Stunden-Algorithmus dann erlaubt, wenn der Test angemessen dafür validiert wurde.

Etwas komplexer ist die Situation bei der im klinischen Alltag etwas weniger kritischen definitiven Diagnose („rulein“). Der Hamburger Algorithmus sah hier so aus, dass ein NSTEMI angenommen wurde, wenn das hsTNI in der Mes-

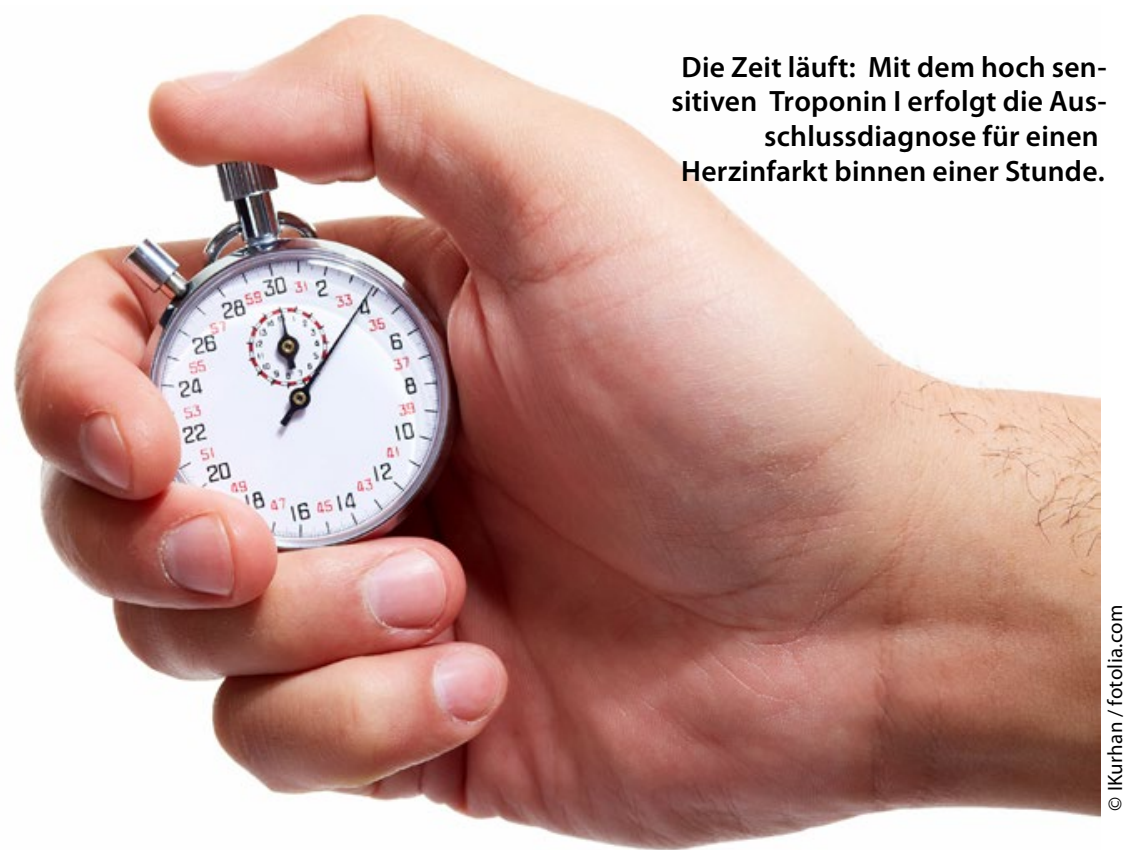

sung sofort nach Aufnahme größer war als $6 \mathrm{ng} / \mathrm{l}$ und innerhalb einer Stunde um mindestens $12 \mathrm{ng} / \mathrm{l}$ anstieg. Dafür wurde ein positiv-prädiktiver Wert für den NSTEMI von $87,1 \%$ (Spezifität 98,0\%) berechnet. In den beiden Validierungskohorten APACE und ADAPT lag der positiv-prädiktive Wert bei $80,4 \%$ bzw. $81,5 \%$.

Diagnostische Grauzone bei $46,7 \%$ Auch das sei nicht schlechter als der Drei-Stunden-Algorithmus, so Westermann. Auf diese Weise kann bereits nach einer Stunde bei 11,9\% der Patienten definitiv ein NSTEMI diagnostiziert und eine Katheteruntersuchung empfohlen werden. Übrig bleiben $46,9 \%$ der Patienten, bei denen sich der akute Infarkt nach einer Stunde durch hsTNI weder definitiv ausschließen noch bestätigen lässt.

Diese Patienten gebe es in ähnlicher Häufigkeit auch beim dreistündigen $\mathrm{Al}$ gorithmus, betonte der Kardiologe. Sie können nicht entlassen werden, sondern erhalten in der Regel die Katheteruntersuchung, um keinen Infarkt zu übersehen. Wie wichtig das ist, zeigte die Auswertung der Sechs-Monats-Mortalität in der BACC-Kohorte: Sie war am größten bei jenen Patienten, bei denen das Troponin keine eindeutige Diagnose oder Ausschlussdiagnose erlaubte.

Philipp Grätzel \section{}

